\title{
EDITORIAL \\ Developing systems of care: association of time to craniectomy with survival in patients with severe combat-related brain injuries
}

\author{
Stacey Quintero Wolfe, MD \\ Department of Neurological Surgery, Wake Forest School of Medicine, Winston-Salem, North Carolina
}

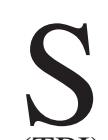
HACKELFORD et al. have provided us with a thoughtful retrospective observational study of mortality associated with combat-related traumatic brain injury (TBI) requiring decompression. ${ }^{13}$ This study identifies a time frame for treatment within which there is a lower mortality, and can be used to help develop systems of care in order to ensure that appropriate resources for stabilization, evacuation, and definitive treatment of combat head injuries for our servicemen and -women are in place.

Shackelford cites the statistic that one-third of battlefield deaths are attributable to TBI, and $9 \%$ of preventable combat deaths are due to TBI, probably due to expanding posttraumatic intracranial hematomas. ${ }^{13}$ While clearly comprising a higher proportion of penetrating and blast injuries than civilian mechanisms, decompression and evacuation of expanding intracranial mass lesions is associated with improved outcomes, and military doctrine over the past decade has shown that emergency craniectomy significantly improves outcome in these patients. ${ }^{14}$

This study examined 213 patients who sustained combat injuries between 2005 and 2015 who underwent craniectomy at deployed surgical facilities. Key to inclusion was evacuation from field care to neurosurgical operative capabilities. In the military, there are 5 "roles" of care, with role 2 consisting of forward resuscitative surgical teams, and role 3 consisting of a fixed facility with forward deployed specialty care (role 1 , mobile forward deployed emergency responder; role 4 , full medical center outside continental US; role 5, full medical center inside continental US). In this study, craniectomy had been performed at role $2(n=63)$ and role $3(n=150)$ facilities.

Postoperative mortality was significantly lower when craniectomy was initiated within 5.33 hours of injury, even after adjusting for between-group differences, including injury severity. Time-dependent Cox models were adjusted for confounders and immortal time bias and the group was divided into 5 subgroups (quintiles) based on time from injury to craniectomy. Quintiles 1-3 showed decreased mortality relative to longer delays (quintiles $4-5$ ) with adjusted hazard ratio $=0.28, p=0.012$. Patients who underwent craniectomy at a role 3 hospital following transfer from a role 2 facility had significantly longer delays to surgery than those who were not transferred, with median delays from injury to craniectomy of 7.1 hours (4.2 prehospital) versus 3.5 hours (2.0 prehospital), respectively $(\mathrm{p}<0.0001)$.

This is the first reported association between time to neurosurgical operative intervention and survival in the combat casualty population. ${ }^{13}$ The 5.33 -hour timeline from injury to craniectomy, beyond which mortality increases, should not be exceeded and ideally will spur even more rapid delivery of care. Given that prehospital delays were the largest variant on outcome, these data will be useful in determining policy to direct battlefield triage, place medical assets, and preplan evacuation to optimize care for our service members. Although there are limitations associated with this type of study, the authors do a good job of detailing those, as well as comparing and contrasting their findings within the framework of existing literature.

The survival rate of military service members injured in combat has significantly improved during the recent decade of military conflict, largely due to advances in trauma care in the prehospital far forward environment. The Defense Health Board circulated a 2015 report summarizing lessons learned from the conflicts in Iraq and Afghanistan and recommendations to preserve this knowledge for future conflicts. ${ }^{4}$ Much of the report focuses on developing systems of care. It was noted that "at the onset of the current conflicts, communication, coordination, and command and control of levels of care and personnel were not 
well coordinated." This is underscored by Shackelford et al. in their findings that prehospital delays from injury to arrival at a craniectomy-capable hospital were $71 \%$ longer on average than in-hospital delays from hospital arrival to the start of neurosurgery. ${ }^{13}$ As in civilian trauma systems, prehospital triage and transport are of key importance to reduce delays when developing systems of care.

Another opportunity for improvement was communication, regarding evacuations as well as inadequate transmission of clinical patient information among levels of care. ${ }^{4}$ Recommendations to provide the global positioning system (GPS) location of the medic in theater, audiovisual telecommunications among military treatment facilities to support situational awareness, and a user-friendly electronic medical documentation system from the field through the various treatment facilities were cited. This is underscored in the current paper, in which the authors state that only 213 (44\%) of 486 craniectomy patients had complete data, although improvement was seen, with injury times decreased from $49 \%$ in $2005-2007$ to $27 \%$ in 2012-2015. ${ }^{13}$

Also highlighted in this report is the need to expand the Department of Defense's partnership with civilian trauma organizations to share information, preserve lessons learned, and improve trauma care. ${ }^{4}$ The Washington committee of the AANS and CNS has supported such legislation as "Mission Zero," which is aimed at achieving zero preventable military deaths by embedding military trauma teams in civilian trauma centers with significant grant funding. While logistically challenging, future consideration should be given to incorporating military medical centers into civilian trauma systems.

Time and treatment capability are important factors for the survival of most critically ill patient populations. Prehospital treatment capability, rapid prehospital transport, and early hospital and surgical treatment capability have been shown to improve trauma outcomes. Ideally, service members with severe TBI will reach neurosurgical capabilities within hours of injury. Military medical doctrine dictates that wounded service members should reach medical care within 4 hours. However, the realities of worldwide military operations may create prohibitive challenges.

Military medical doctrine advocates for effective medical capability at or near the anticipated point of injury, optimizing time to treatment and subsequently patient survival. The spirit of this doctrine applies to civilian systems of care such as trauma, ST-elevation myocardial infarction (STEMI), and our newly developing stroke algorithms. In stroke, there has been considerable concern, change, and controversy in developing stroke systems of care since the Brain Attack Coalition recommendation for Stroke Center designation in 2000. Following the randomized studies in 2015 dictating thrombectomy as the standard of care, $2,3,5,6,12$ expansion of the intervention window up to 24 hours for patients with preserved penumbra, ${ }^{1,8}$ new stroke guidelines, ${ }^{10}$ partial guidelines retraction, and the newest World Federation of Interventional and Therapeutic Neuroradiology consensus recommendations, ${ }^{9}$ the medical community has been quickly changing and adapting triage and transportation protocols. The question of bypass- ing a primary stroke center or whether to allow care in lower-volume thrombectomy-capable centers are important questions. There is mounting evidence that there is a clear volume-outcome relationship. Rinaldo et al. $(\mathrm{N}=$ 8500 over 4 years) showed mortality rates to be significantly lower at high-volume centers $(9.8 \%)$ compared to medium-volume $(14.9 \%)$ or low-volume $(19.7 \%)$ centers. ${ }^{11}$ They also showed that the benefit of treatment at a highvolume center may outweigh any detrimental consequences from transfer delays. New recommendations suggest minimums of 50 thrombectomies annually to maintain competence. ${ }^{9}$ These data are important when developing systems of care for any time-sensitive medical condition.

Shackelford et al.'s report ${ }^{13}$ reinforces the importance of reducing time to definitive treatment, transporting to the highest possible level of care while risk-adjusting travel time, and improving and standardizing leadership and communication. The call to arms must be made to place patient care above insurance or hospital systems; ensure that our Emergency Medical Services colleagues have adequate resources and protocols; ensure that definitive care is provided and directed by specialty-specific physicians with high-quality, regulated training in a high-volume system with transparent outcome data; and ensure that data acquisition is supported and made easier by federal mandates regarding interoperability of electronic medical record systems. Continued optimization of systems of care is a requirement for both our military and civilian populations.

https://thejns.org/doi/abs/10.3171/2018.9.FOCUS18497

\section{References}

1. Albers GW, Marks MP, Kemp S, Christensen S, Tsai JP, Ortega-Gutierrez S, et al: Thrombectomy for stroke at 6 to 16 hours with selection by perfusion imaging. N Engl J Med 378:708-718, 2018

2. Berkhemer OA, Fransen PS, Beumer D, van den Berg LA, Lingsma HF, Yoo AJ, et al: A randomized trial of intraarterial treatment for acute ischemic stroke. N Engl J Med 372:11-20, 2015

3. Campbell BC, Mitchell PJ, Kleinig TJ, Dewey HM, Churilov L, Yassi N, et al: Endovascular therapy for ischemic stroke with perfusion-imaging selection. N Engl J Med 372:10091018, 2015

4. Defense Health Board: Combat Trauma Lessons Learned From Military Operations of 2001-2013. Arlington, VA: Office of the Assistant Secretary of Defense Health Affairs, 2015 (http://www.dtic.mil/dtic/tr/fulltext/u2/1027320.pdf) [Accessed October 3, 2018]

5. Goyal M, Demchuk AM, Menon BK, Eesa M, Rempel JL, Thornton J, et al: Randomized assessment of rapid endovascular treatment of ischemic stroke. N Engl J Med 372:1019_ 1030,2015

6. Jovin TG, Chamorro A, Cobo E, de Miquel MA, Molina CA, Rovira A, et al: Thrombectomy within 8 hours after symptom onset in ischemic stroke. N Engl J Med 372:2296-2306, 2015

7. Kotwal RS, Staudt AM, Trevino JD, Valdez-Delgado KK, Le TD, Gurney JM, et al: A review of casualties transported to role 2 medical treatment facilities in Afghanistan. Mil Med 183 (Suppl 1):134-145, 2018

8. Nogueira RG, Jadhav AP, Haussen DC, Bonafe A, Budzik RF, Bhuva P, et al: Thrombectomy 6 to 24 hours after stroke 
with a mismatch between deficit and infarct. N Engl J Med 378:11-21, 2018

9. Pierot L, Jayaraman MV, Szikora I, Hirsch JA, Baxter B, Miyachi S, et al: Standards of practice in acute ischemic stroke intervention: international recommendations. J Neurointerv Surg [epub ahead of print], 2018

10. Powers WJ, Derdeyn CP, Biller J, Coffey CS, Hoh BL, Jauch EC, et al: 2015 American Heart Association/American Stroke Association focused update of the 2013 Guidelines for the Early Management of Patients With Acute Ischemic Stroke Regarding Endovascular Treatment: a guideline for healthcare professionals from the American Heart Association/American Stroke Association. Stroke 46:3020-3035, 2015

11. Rinaldo L, Brinjikji W, Rabinstein AA: Transfer to high-volume centers associated with reduced mortality after endovascular treatment of acute stroke. Stroke 48:1316-1321, 2017

12. Saver JL, Goyal M, Bonafe A, Diener HC, Levy EI, Pereira VM, et al: Stent-retriever thrombectomy after intravenous t-PA vs. t-PA alone in stroke. N Engl J Med 372:2285-2295, 2015
13. Shackelford SA, del Junco DJ, Reade MC, Bell R, Becker T, Gurney J, et al: Association of time to craniectomy with survival in patients with severe combat-related brain injury. Neurosurg Focus 45(6):E2, 2018

14. Weisbrod AB, Rodriguez C, Bell R, Neal C, Armonda R, Dorlac W, et al: Long-term outcomes of combat casualties sustaining penetrating traumatic brain injury. J Trauma Acute Care Surg 73:1525-1530, 2012

\section{Disclosures}

The author reports no conflict of interest.

\section{Correspondence}

Stacey Quintero Wolfe: sqwolfe@wakehealth.edu.

INCLUDE WHEN CITING

DOI: 10.3171/2018.9.FOCUS18497. 